\title{
Fabrication of Porous Aluminum Coating by Cored Wire Arc Spray for Anchoring Antifouling Hydrogel Layer
}

\author{
Jianxin Wen ${ }^{1,2,3} \cdot$ Ziheng Song ${ }^{1,2,3} \cdot$ Xiuyong Chen ${ }^{1,3} \cdot \mathrm{Hua} \mathrm{Li}^{1,3}$
}

Submitted: 12 May 2021 / in revised form: 27 July 2021 / Accepted: 30 July 2021 / Published online: 18 August 2021

(C) ASM International 2021

\begin{abstract}
Biofouling has been persisting as a worldwide problem due to the difficulties in finding efficient environment-friendly antifouling coatings for long-term applications. Developing novel coatings with desired antifouling properties has been one of the research goals for surface coating community. Recently hydrogel coating was proposed to serve as antifouling layer, for it offers the advantages of the ease of incorporating green biocides, and resisting attachment of microorganisms by its soft surface. Yet poor adhesion of the hydrogel on steel surfaces is a big concern. In this study, porous matrix aluminum coatings were fabricated by cored wire arc spray, and the sizes of the pores in the aluminum ( $\mathrm{Al}$ ) coatings were controlled by altering the size of the cored powder of sodium chloride.
\end{abstract}

This article is an invited paper selected from presentations at the 2021 International Thermal Spray Conference, ITSC2021, that was held virtually May 25-28, 2021 due to travel restrictions related to the coronavirus (COVID-19) pandemic. It has been expanded from the original presentation.

\section{Xiuyong Chen}

chenxiuyong@nimte.ac.cn

$凶$ Hua Li

lihua@nimte.ac.cn

1 Key Laboratory of Marine Materials and Related Technologies, Ningbo Institute of Materials Technology and Engineering, Chinese Academy of Sciences, Ningbo 315201, China

2 Center of Materials Science and Optoelectronics Engineering, University of Chinese Academy of Sciences, Beijing 100049, China

3 Zhejiang Engineering Research Center for Biomedical Materials, Cixi Institute of Biomedical Engineering, Ningbo Institute of Materials Technology and Engineering, Chinese Academy of Sciences, Ningbo 315201, China
Silicone hydrogel was further deposited on the porous coating. The hydrogel penetrated into the open pores of the porous $\mathrm{Al}$ coatings, and the porous $\mathrm{Al}$ structure significantly enhanced the adhesion of the hydrogel. In addition, hydrogel coating exhibited very encouraging antifouling properties.

Keywords antifouling adhesion - cored wire arc spraying $\cdot$ hydrogel $\cdot$ porous coating

\section{Introduction}

As one of the major problems for marine infrastructures, biofouling is caused by the attachment of microorganisms when materials are immersed in seawater. Biofouling usually involves three consecutive stages, adsorption of some organics like polysaccharides and proteins on the surface to form conditioning layer, formation of biofilm through attachment and colonization of bacteria, diatoms and other microorganisms on the conditioning layer and secrete extracellular polymeric substances (EPS), and promoted settlement of larvae of fouling organisms to form biofouling (Ref 1). Serious impacts of biofouling have been extensively reported, for example accelerated corrosion (Ref 2), causing significant economic loss (Ref 3). Many antifouling strategies have been developed in recent decades and construction of surface coating containing biocides is the most effective method (Ref 4-6). However, the release of biocides is dangerous for other marine organisms, triggering worldwide concerns. Tributyltin (TBT) was banned by the International Maritime Organization (IMO) in order to avoid environmental issues in 2008 (Ref $7,8)$. In addition to the release of biocides, antifouling methods are facing high costs and a series of production 
issues from the laboratory to industrialization (Ref 9). Developing environmental-friendly antifouling coating with the ease of large-scale fabrication is therefore essential for marine structures combating biofouling.

Hydrogel is a porous and soft polymer material, and has been extensively investigated for potential applications for drug delivery and sensors (Ref 10-4). In recent years, excellent antifouling properties were also reported for hydrogels like polyethylene glycol (PEG) and polyvinyl alcohol (PVA) hydrogel (Ref 15, 16) due to their low surface energy and floppy nature. According to Brady (Ref 17), adhesion of fouling species was correlated to the square root of multiplication of elastic modulus (E) and surface free energy $(\gamma)$. Another research indicated that hydrogel was dynamic in seawater because of the low elastic modulus (Ref 18), offering the difficulties for microorganisms to attach on the surface of materials. Bressy et al. (Ref 19) reported that surface with low elastic modulus provided an easy-release path for microorganisms. Marine organisms require less energy detaching from low elastic modulus surfaces than that from high elastic modulus surfaces. Meanwhile, according to a previous report (Ref 20), when the surface free energy of the material is between $20-30 \mathrm{~mJ} / \mathrm{m}^{2}$, the minimum strength of biological adhesion to substrates is always found. However, the hydrogel coating can be easily damaged because of weak adhesion (Ref 21). Efforts have therefore been made to improve the hydrogel adhesion for long-term applications. Among the attempted techniques, functionalization of the hydrogel coating with specific functional groups could provide the capability to form chemical bonding with substrate. For example, catechol can form covalent and non-covalent bonds with many functional groups (Ref 22), which is a good adhesive for hydrogel. Westwood et al. (Ref 23) found that hydrogel modified with catechol functional groups strongly attached to a variety of substrate surfaces. Moreover, Yang et al. (Ref 24) used epoxy as the bond-coat to improve the binding force of hydrogel and the hydrogel exhibited excellent antifouling property. However, the synthesis of hydrogel with specific functional groups requires strict control of the reaction conditions and may involve the use of toxic reagents (Ref 25). Alternatively physical adhesion by which hydrogel and porous substrate could form mechanical interlocking only needs porous substrate, and the fixation of hydrogel opts for rough surfaces of the matrix (Ref 26). Construction of matrix substrate with controllable porous structures for anchoring antifouling hydrogel layers is therefore essentially required.

To date, many techniques have been attempted for preparing porous structures, such as electrodeposition (Ref 27), microphase separation (Ref 28), and 3D printing (Ref 29). However, application of the abovementioned methods for large-scale construction of porous structures is questionable. Exploring cost-efficient fabrication strategies is highly desirable. As one of the most important surface techniques, thermal spraying especially arc spraying has been widely used in industry due to its low cost, simple process, and high efficiency. However, there are few reports on the preparation of porous coatings by thermal spraying (Ref 30,31) for improving the adhesion of the antifouling layers. Here, we report a controllable porous aluminum coating by cored wire arc spraying that was sealed with antifouling hydrogel layer, exceptionally enabling enhancements in both physical adhesion of hydrogel layers and antifouling properties of the thermal sprayed coatings. Specifically, sodium chloride of different sizes as a pore-forming agent was used to prepare different sizes porous aluminum ( $\mathrm{Al}$ ) coatings by cored wire arc spraying. Then silicone hydrogel was brushed on the porous Al coatings. The physical adhesion of the silicone hydrogel layers on the coatings, antifouling properties and corrosion resistance of the coatings were investigated.

\section{Materials and Methods}

\section{Sample Preparation}

Coatings were fabricated by a twin-wire arc spray system (EuTronic Arc Spray $4 \mathrm{HF}$, Castolin-Eutectic Pte Ltd., Nashville, USA). A commercially available sodium chloride powder (Aladdin, China) was used as filler of Al-based cored wire. The planetary mill (QM-3SP4, China) was used to reduce the particle size of the as-received sodium chloride powder. After ball milling, the powder was sieved. The average particle size of the powder was obtained based on field emission scanning electron microscopy (FESEM) (Regulus SU8230, Japan) images. The Al coating and three different porous aluminum coatings were prepared in this study. For the Al coating, the samples were prepared by non-cored wire arc spray. For the porous Al coatings (namely Coating-100, Coating-200, Coating-500), the sizes of the pores in the $\mathrm{Al}$ coating were controlled by altering the size of the cored powder with $\sim 100 \mu \mathrm{m}, \sim 200 \mu \mathrm{m}$ and $\sim 500 \mu \mathrm{m}$, respectively. The width and thickness of the pure aluminum strip (Dongguan Hualv Aluminum Co., Ltd, China) used to produce the cored wire was $7 \mathrm{~mm}$ and $300 \mu \mathrm{m}$, respectively. 316 stainless steel plates (Wanren Stainless Steel, China) with the dimension of $20 \times 20 \times 3$ $\mathrm{mm}^{3}$ were used as the substrates. The substrates were grit blasted and ultrasonically cleaned in alcohol before deposition. The current and voltage of arc spraying were $150 \mathrm{~A}$ and $36 \mathrm{~V}$, respectively, and the spray distance was $200 \mathrm{~mm}$. The pressure of compressed air was 0.6 MPa. After the spraying, the sodium chloride in the coatings was dissolved 
by $2 \mathrm{~mol} \cdot \mathrm{L}^{-1}$ hydrochloric acid and deionized water to form porous coating structure. The silicone hydrogel (Zhejiang Marine Development Research Institute, China) was finally brushed on the porous coatings for antifouling property. The fabrication diagram of the silicone hydrogel coating is shown in Fig. 1.

\section{Algae Cultivation and Adhesion Testing}

Artificial seawater (ASW) was prepared according to ASTM D1141-98 and sterilized for $20 \mathrm{~min}$ at $121{ }^{\circ} \mathrm{C}$. Chlorella sp. (obtained from Ningbo University, China) was cultivated in ASW with Guillard's F/2 medium. The Chlorella sp. was cultured in an incubator at $22{ }^{\circ} \mathrm{C}$ with a light/dark cycle of $12 \mathrm{~h}: 12 \mathrm{~h}$. The hydrogel coatings, the as-sprayed $\mathrm{Al}$ coatings and the polished $\mathrm{Al}$ coatings were cleaned with alcohol ultrasonic and then deionized water before putting into 6 -well plates with algae suspension. The samples were immersed in algae suspension for 1, 3, 5, 7 days and three samples were taken from each group as parallel samples for each test. ASW was then used to wash the unattached algae. The adhesion of Algae was examined by confocal laser scanning microscopy (CLSM, Leica TCS SP5, Germany) after immersed in $2.5 \%$ glutaraldehyde for $2 \mathrm{~h}$. The adhesion ratio of algae refers to the area fraction covered by Chlorella sp.. Coverage of Chlorella sp. on the coatings was obtained by image software ImageJ (Version: $1.47 \mathrm{v}$ ) analysis. The adhesion ratio value was acquired based on the average of three measurements. All reagents were purchased from Aladdin (Aladdin, China) and used as received without further purification.

\section{Sample Characterization}

Phase composition of powders and coatings were determined by x-ray diffraction (XRD) (D8 Advance Davinci, Germany) operated at $40 \mathrm{kV}$ and $40 \mathrm{~mA}$ with $\mathrm{Cu} \mathrm{Ka}$ radiation. General Structure Analysis System (GSAS) and EXPGUI software package was used to perform Rietveld analysis (Ref 32). Morphologies of the raw powders and the coatings were characterized by SEM. The filling ratio refers to the proportion of the powders filling inside the cored wire, and the following Eq (1) was applied for calculation:

Filling ratio $=\frac{M_{T}-M_{S}}{M_{T}} \times 100 \%$

where $M_{T}, M_{S}$ is the total mass of cored wire and the mass of the cored wire after removing the filling powders, respectively. The filling ratio value was obtained based on the averaging of three measurements. The SEM images (planar surface view) of the polished coatings were analyzed using ImageJ (Version 1.47) to calculate their porosities. Five measurements were conducted in this study. The adhesion of silicone hydrogel on porous $\mathrm{Al}$ coating was assessed according to ASTM-C663-01(Ref 33) by universal testing machine (SANS CMT 5205, China). In this study, stainless steel rods (25 $\mathrm{mm}$ in diameter) were bonded to the sample. A tensile stress was then applied at a speed of $1 \mathrm{~mm} / \mathrm{min}$. The adhesion strength value was obtained based on the average of three measurements. The data of adhesion strength were expressed as mean \pm standard deviation (SD) for $\mathrm{n}=3$. Statistical analysis was conducted with software OriginPro (version 7.5) at confidence levels of $99.5 \%$. Surface energy of the hydrogel coatings was obtained by contact angle measurement (Dataphysics OCA25, Germany) based on the OwensWendt-Rabel-Kaelble (OWRK) (Ref 34). $2 \mu \mathrm{L}$ of distilled water and ethanol was used as the probe liquid. The compression test was performed to obtain the elastic modulus of the hydrogel using a universal testing machine (SUST CMT 1104, China) (Ref 35). The samples with a 10 $\mathrm{mm}$ diameter and $5 \mathrm{~mm}$ height were used for the compression tests. The compression rate was set at $20 \mathrm{~mm} / \mathrm{min}$ and the test stopped when the compression reached $60 \%$ and selected the slope of the linear elastic within $10 \%$ of the strain as elastic modulus. The elastic modulus value was obtained based on the average of three measurements.

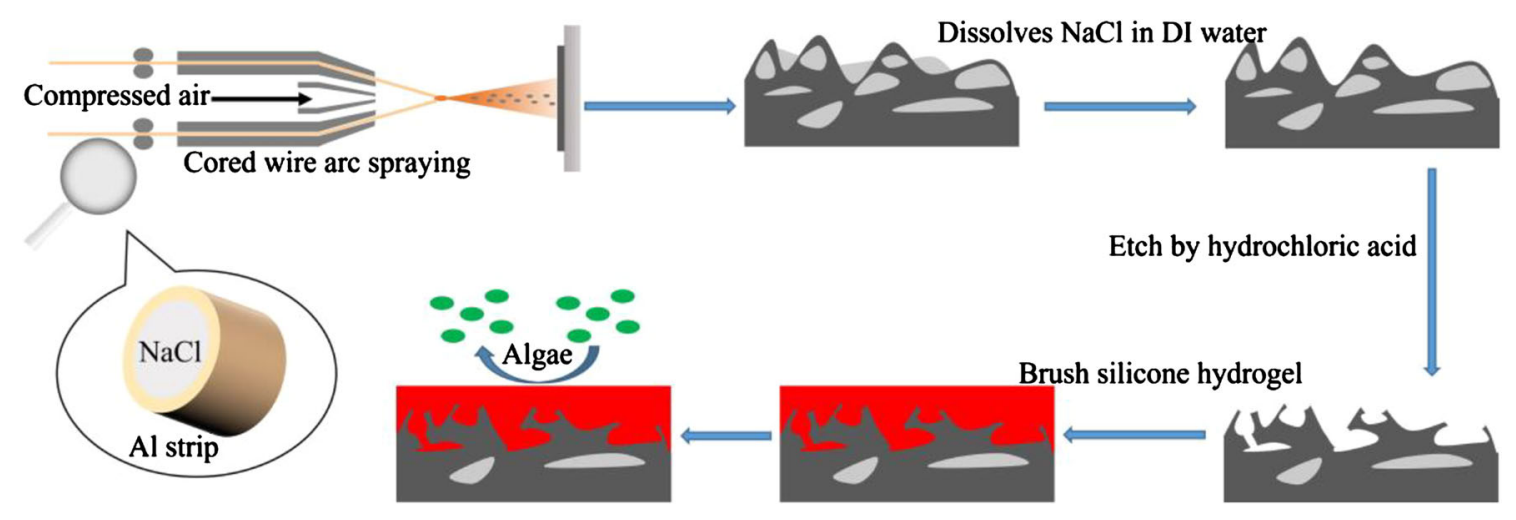

Fig. 1 Fabrication diagram of the silicone hydrogel coating 
Corrosion tests were performed via a three-electrode system (Ref 36) (Chenhua CHI-660E, Shanghai, China). The saturated calomel electrode was used as the reference electrode and platinum was used as the counter electrode. The work electrode was immersed in ASW for $2 \mathrm{~h}$ to acquire the stable open circuit potential $\left(E_{\text {ocp }}\right)$. Potentiodynamic polarization curves were obtained with potential range from $E_{\text {ocp }}+500 \mathrm{mV}$ to $E_{\text {ocp }}-500 \mathrm{mV}$. The Tafel curve parameters (Ref 37 ) including corrosion potential $\left(E_{\text {corr }}\right)$, corrosion current $\left(I_{\text {corr }}\right)$, cathode Tafel slope $\left(\beta_{c}\right)$, anode Tafel slope $\left(\beta_{a}\right)$ were obtained by nova 2.1 software. Each group had at least three specimens to ensure repeatability.

\section{Results and Discussion}

Three different sizes of sodium chloride powders were used as pore-forming agent (Fig. 2). Figure 2a and b show the morphology of the powder of sodium chloride after ball milling with irregular shapes. The average size of the three powders was $\sim 100 \mu \mathrm{m}, \sim 200 \mu \mathrm{m}$ and $\sim 500 \mu \mathrm{m}$, respectively. Porous matrix $\mathrm{Al}$ coatings (namely, Coating100, Coating-200, Coating-500) were fabricated by cored wire arc spray, and the sizes of the pores in the $\mathrm{Al}$ coatings were controlled by altering the size of the cored powder of sodium chloride. Figure 3 shows the XRD pattern of the $\mathrm{Al} / \mathrm{NaCl}$ composite coatings (Fig. 3a) and porous $\mathrm{Al}$ coatings (Fig. 3b). The XRD spectra of the three $\mathrm{Al} / \mathrm{NaCl}$ composite coatings show the peaks of sodium chloride, indicating that the $\mathrm{Al} / \mathrm{NaCl}$ composite coatings were successfully prepared by cored wire arc spraying. Rietveld analysis showed that percentage of the $\mathrm{NaCl}$ content in all three coatings was 3.9wt.\% (Coating-100), 4.4wt.\% (Coating-200) and 4.5wt.\% (Coating-500), respectively. It is worth noting that the $\mathrm{NaCl}$ content in the three coatings was slightly different which is probably due to the uneven distribution of $\mathrm{NaCl}$ in the coatings. No peak of $\mathrm{NaCl}$ was observed in the porous $\mathrm{Al}$ coatings after dissolving sodium chloride. The XRD pattern did not show any other impurity peaks. However, elemental analysis by EDS showed that $1.67 \mathrm{wt} . \%$ oxygen was present in the Al coating. The phenomenon that no $\mathrm{Al}_{2} \mathrm{O}_{3}$ XRD peak was detected in the coating is probably due to the relatively low content of $\mathrm{Al}_{2} \mathrm{O}_{3}$ in the $\mathrm{Al}$ coating which is below the XRD detection limit. Similar results were previously reported (Ref 38-40).

Surface morphologies of the porous Al coatings are shown in Fig. 4. Typical surface morphology of the $\mathrm{Al}$ coating is observed (Fig. 4a). It can be seen that there are many small pores on the surface of the $\mathrm{Al}$ coating after $\mathrm{HCl}$ etching (Fig. $4 \mathrm{~b}$ and c). The pore size of the porous coatings increased as the particle size of $\mathrm{NaCl}$ increased (Fig. 4c-e). It is worth noting that the pore size of the porous coatings is smaller than that of the original $\mathrm{NaCl}$ powders, which may be due to the fact that $\mathrm{NaCl}$ powder was atomized or broken in the high-speed air flow during the spraying process (Ref 41, 42). The pores within the porous Al coating are irregular, which is likely due to the irregular shape of the $\mathrm{NaCl}$ particles. In order to understand the adhesion strength between the silicone hydrogel and the matrix Al coatings, the cross-sectional morphology and Energy Dispersive x-ray Spectroscopy (EDS) of interface between the silicone hydrogel and the porous $\mathrm{Al}$ coatings were investigated, as shown in Fig. 5, where the green area is $\mathrm{Si} \mathrm{K} \alpha 1$ (assigned to the silicone hydrogel), and the red area is $\mathrm{Al} \mathrm{K} \alpha 1$ (refers to the $\mathrm{Al}$ coating). The silicone hydrogel is tightly anchored to the porous aluminum coating. It could be seen that the silicone hydrogel is completely immersed into the pores of the porous $\mathrm{Al}$ coating. Additionally, smaller secondary pores (the area pointed by the white arrow) were observed in the Coating200 and the Coating-500, and the silicone hydrogel also infiltrated into the secondary pores, which probably facilitate the adhesion of silicone hydrogel on the matrix $\mathrm{Al}$ coating. However, only fewer pores were observed in the Coating-100, and no obvious secondary pores were found. It is similar to the interface morphologies between the $\mathrm{Al}$ coating and the silicone hydrogel.

Figure 6 shows the porosities of the coatings. A relatively dense $\mathrm{Al}$ coating with a porosity of $\sim 1.5 \%$ was
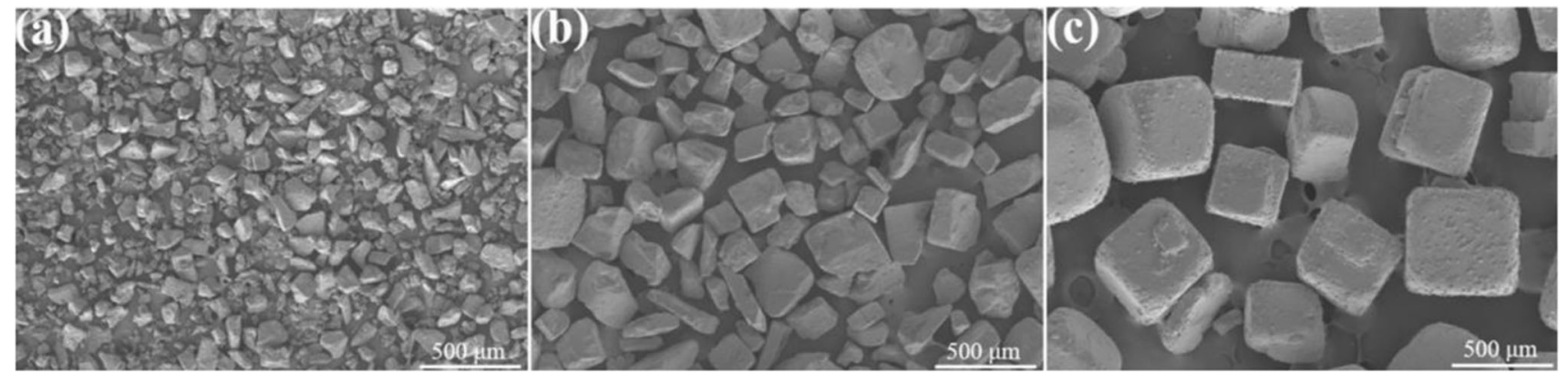

Fig. 2 The surface morphology of the three $\mathrm{NaCl}$ powders with the size of (a) $\sim 100 \mu \mathrm{m}$, (b) $\sim 200 \mu \mathrm{m}$, and (c) $\sim 500 \mu \mathrm{m}$ 
Fig. 3 XRD patterns of the coatings before (a) and after (b) the $\mathrm{NaCl}$ particles were removed
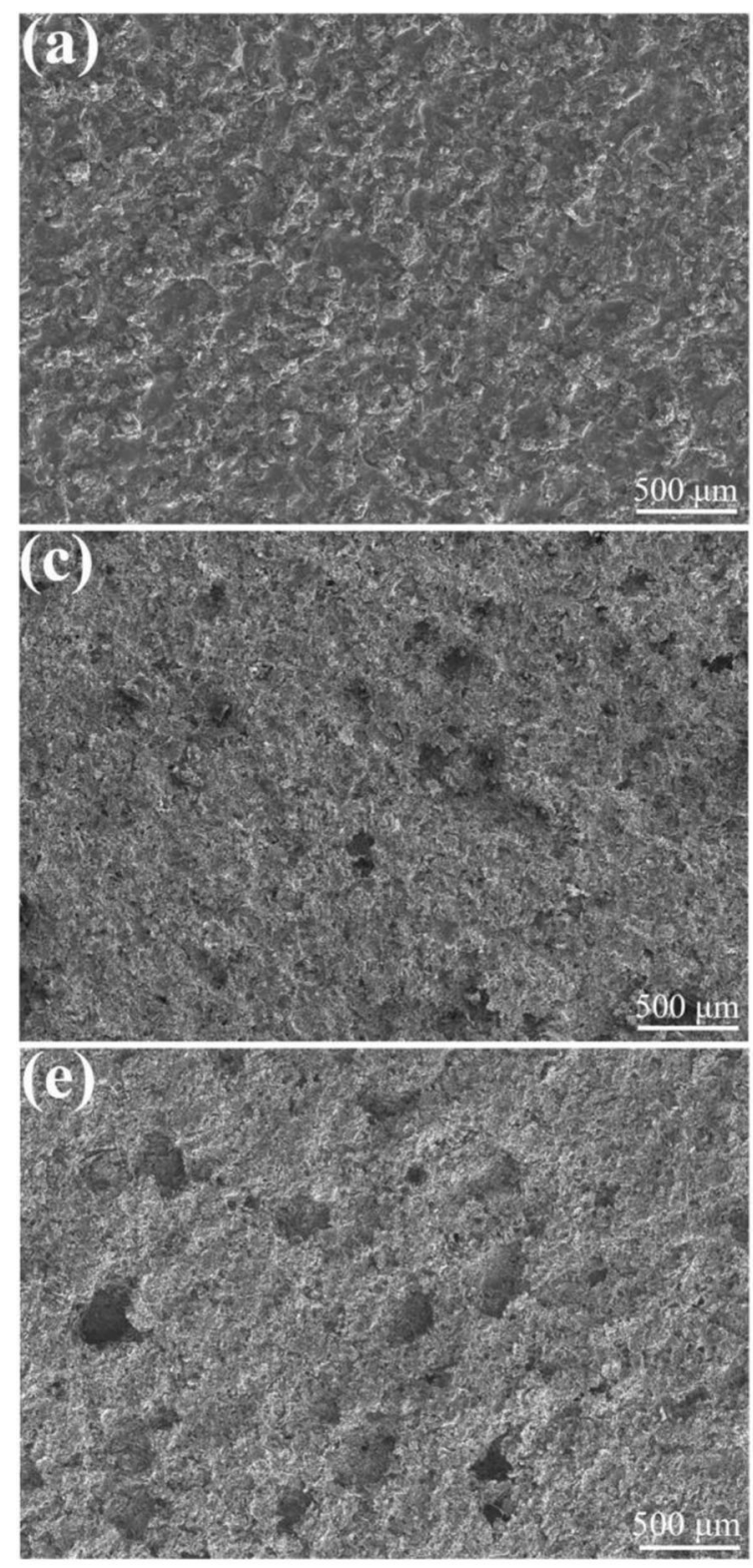

Fig. 4 Surface morphologies of the coatings: (a) Al coating, (b) Al coating after $\mathrm{HCl}$ etching, (c) Coating-100, (d) Coating-200, and (e) Coating500
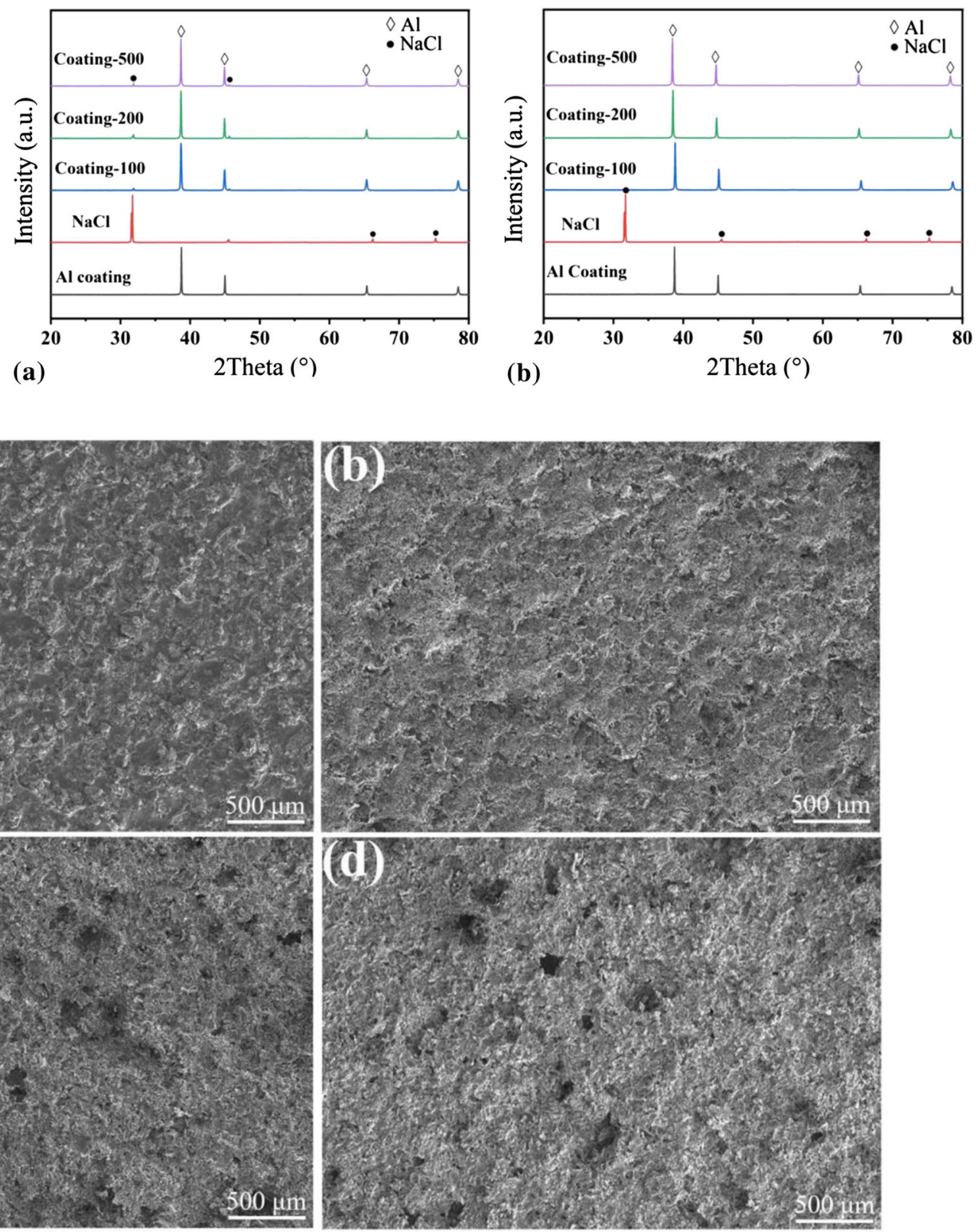


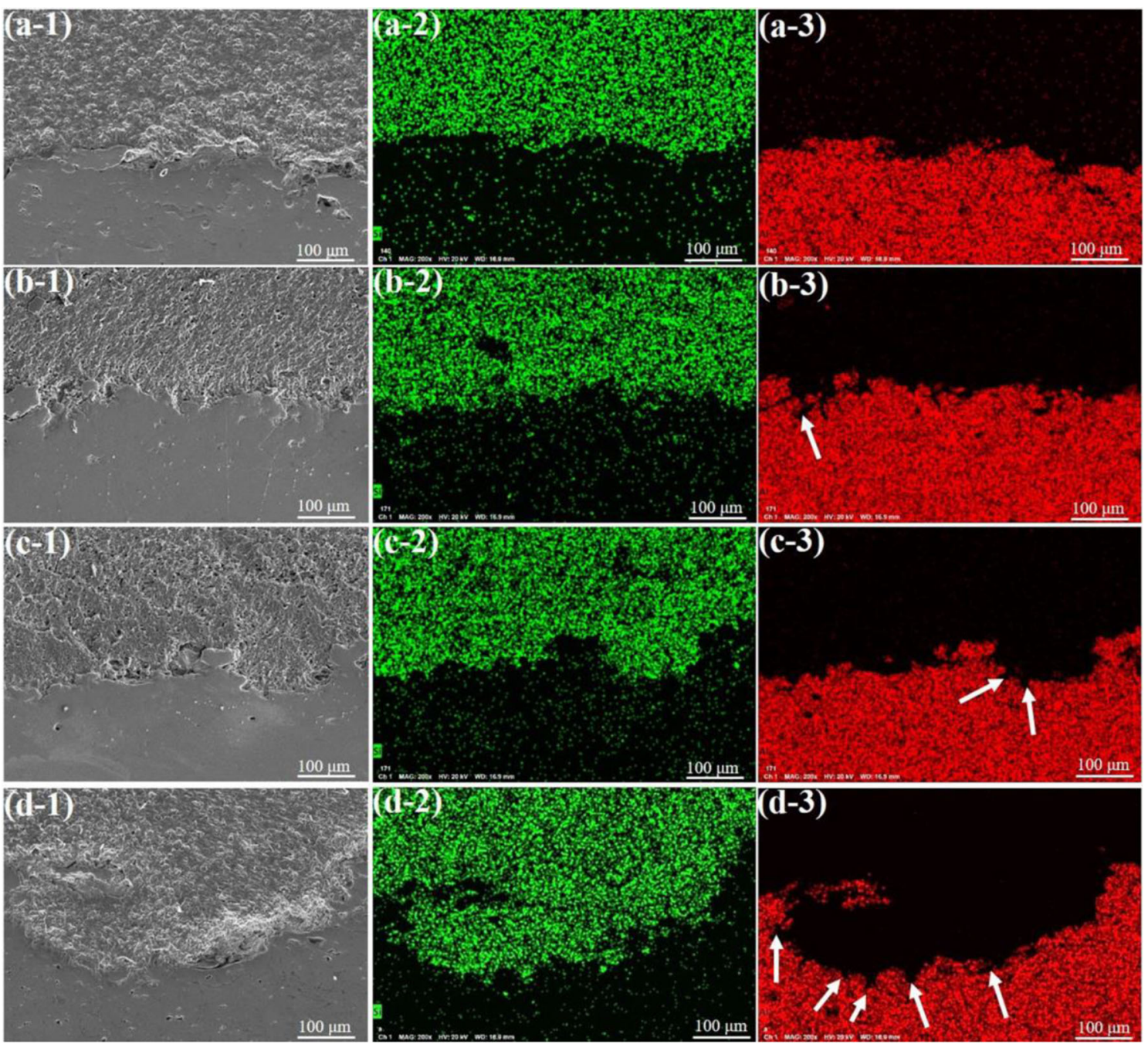

Fig. 5 SEM and EDS mapping of the interface between the silicone hydrogel and the porous Al coatings: (a) the Al coating, (b) the Coating-100, (c) the Coating-200, and (d) the Coating-500; (-1) is the

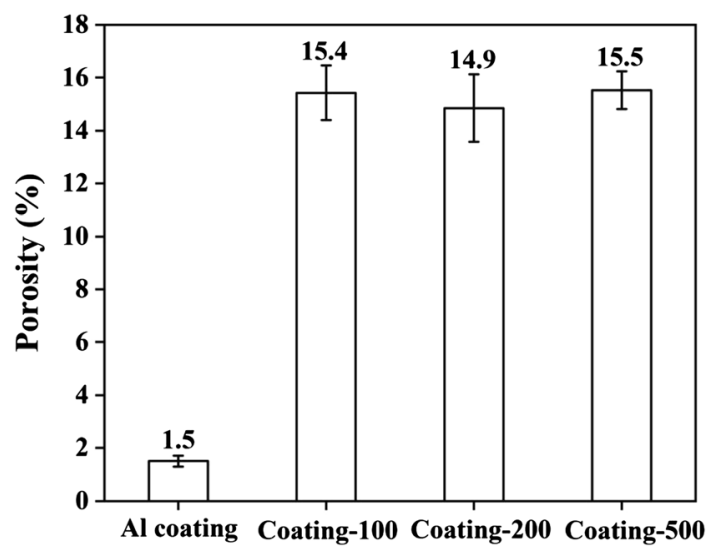

Fig. 6 The porosity of the $\mathrm{Al}$ coating and three porous $\mathrm{Al}$ coatings

prepared by arc spraying, while the porosities of the three porous coatings were greatly increased with porosities of cross-sectional morphology of the coatings; (-2) is the EDS mapping of $\mathrm{Si} \mathrm{K} \alpha 1 ;(-3)$ is the EDS mapping of $\mathrm{Al} \mathrm{K} \alpha 1$. The areas pointed by the white arrows are the smaller secondary pores

$\sim 15.4 \%, \sim 14.9 \%$ and $\sim 15.5 \%$, respectively. It is worth noting that no significant difference was observed among the three porous coatings. This is because the filling rate of the three powder core wires $(\sim 35.8 \%, \sim 35.7 \%$ and $\sim 36.5 \%$, respectively) is about the same. The adhesion strength between the silicone hydrogel and the Al coating was obtained by tensile test, as shown in Fig. 7. The tensile strength increased as the size of the cored powder of sodium chloride increased. The adhesion of silicone hydrogels on the Coating-200 was slightly improved than that of the Al coating and the Coating-100. The Coating500 showed the highest tensile strength value. Interestingly, the porosity of the three porous coatings is similar, but the silicone hydrogel adhesion is quite different. A coating with hierarchical and deeper pores had better adhesion because of the increased contact area between the silicone hydrogel and the matrix coating, and the silicone 


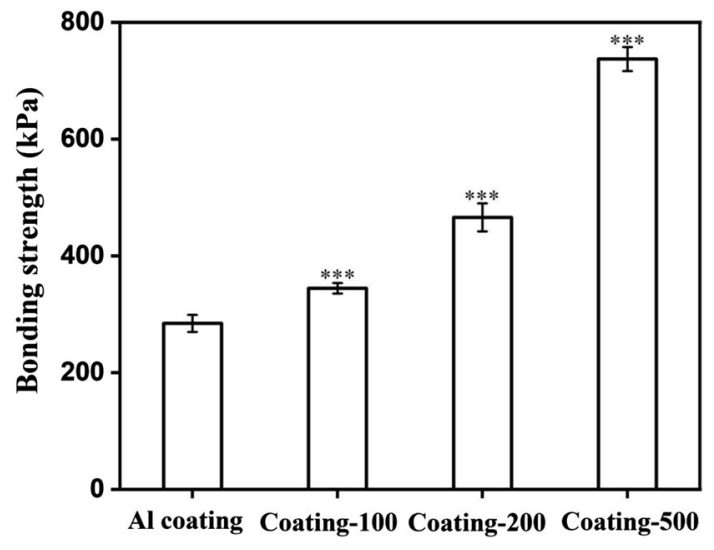

Fig. 7 The adhesion strength of silicone hydrogel on the Al coating and the three porous $\mathrm{Al}$ coatings. Error bars represent mean \pm SD for $\mathrm{n}=3$. $* * * \mathrm{p}<0.005$ compared with $\mathrm{Al}$ coating hydrogel infiltrated in the porous structure to form a mechanical interlock, which could be seen from Fig. 5. The result is consistent with a previous research [43]. Figure 8 shows the cross-sectional and surface morphologies of the coatings after tensile tests. It could be seen that some silicone hydrogels are still attached to the matrix coating surfaces (the area pointed by the white arrow). Therefore, part of the fracture occurred between the matrix coating and the silicone hydrogel, and part of the fracture occurred in the silicone hydrogel during the tensile process.

Figure 9 shows the $\mathrm{Al}$ coating, the polished $\mathrm{Al}$ coating, and the silicone hydrogel coating soaked in the Chlorella suspension for $1,3,5$, and 7 days to evaluate the antifouling properties of the coatings. The polished $\mathrm{Al}$ coating with a flat surface was used for antifouling testing in order to eliminate the influence of roughness on algae adhesion. The adhesion rate of Chlorella was calculated by
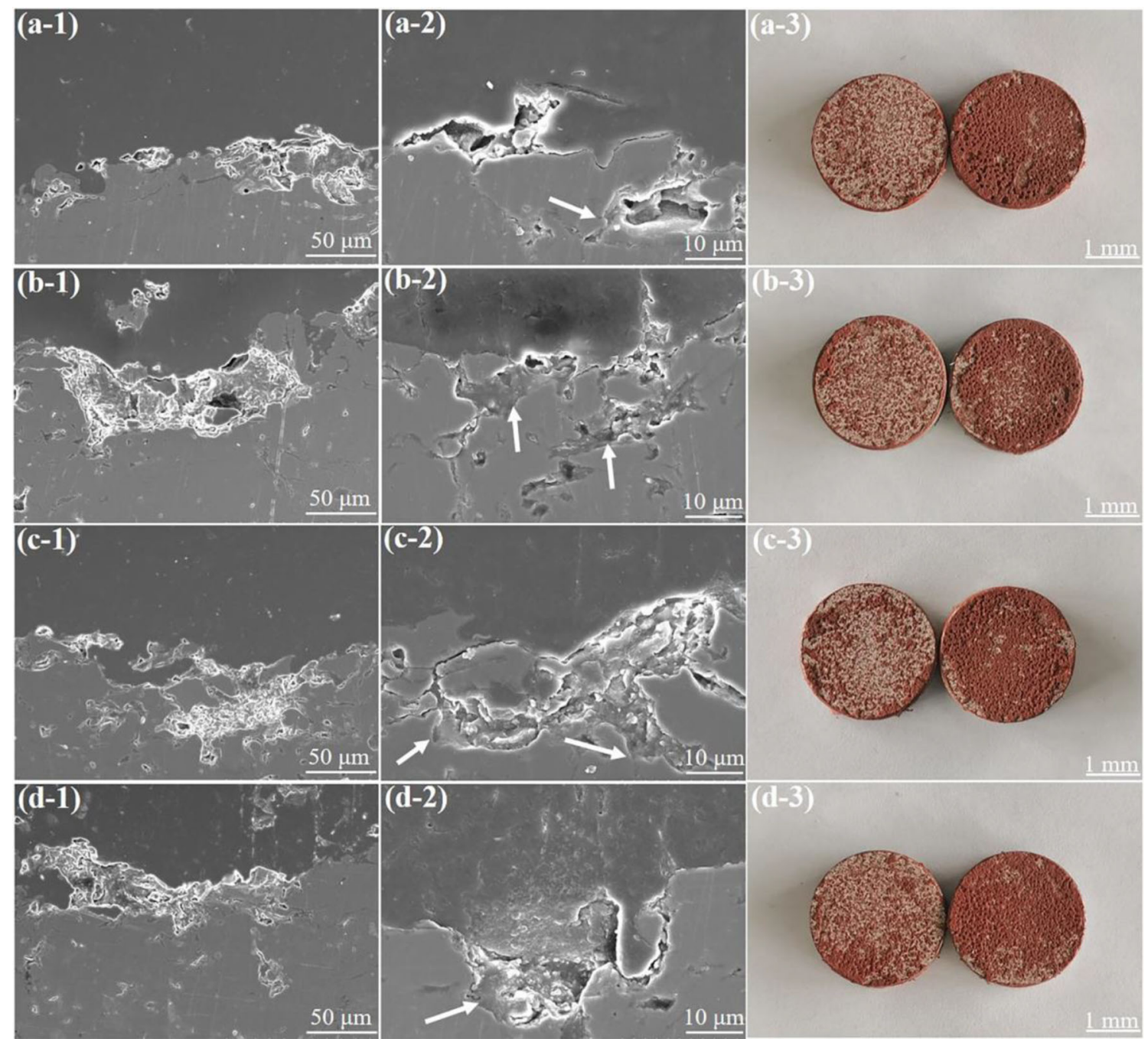

Fig. 8 Cross-sectional view of the coatings after tensile tests: (a) Al coating, (b) Coating-100, (c) Coating-200, and (d) Coating-500. (-2 is magnified view of the cross-sectional morphology of the coatings. -3 is the fractographic image of the ruptured surfaces obtained by digital camera.) The areas pointed by the white arrows are the interface between silicone hydrogel and porous $\mathrm{Al}$ coating 


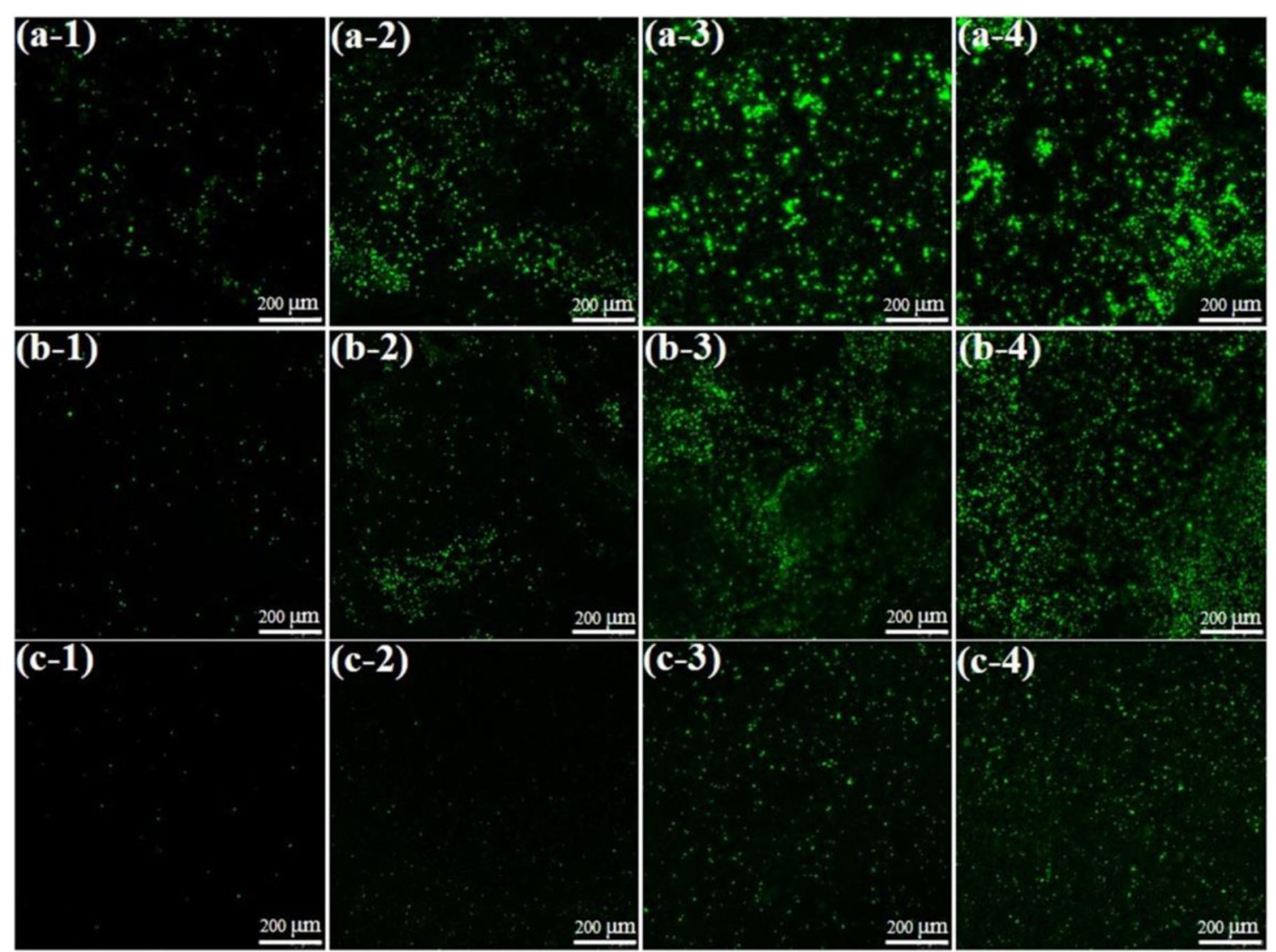

Fig. 9 CLSM images of the Chlorella adhered on the coating surfaces after incubation for 1(-1), 3(-2), 5(-3), and 7(-4) days: (a) the Al coating, (b) the polished Al coating, and (c) the silicone hydrogel coating. Scale bars: $200 \mu \mathrm{m}$

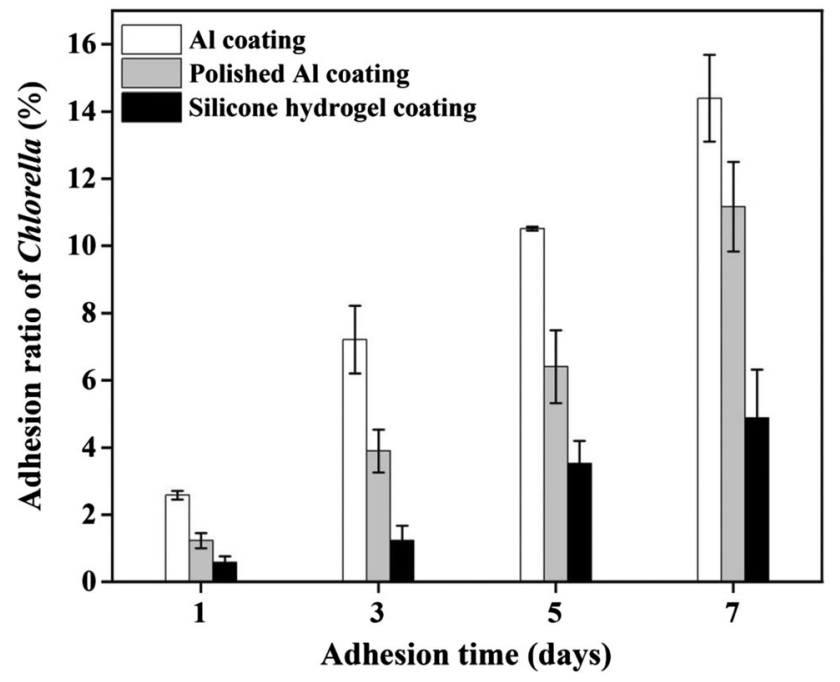

Fig. 10 The adhesion ratio of Chlorella on the three coatings after different incubation time

ImageJ, as presented in Fig. 10. It can be seen that the adhesion rate of algae was related to the immersion time. As the immersion time increases, more algae adhered to the

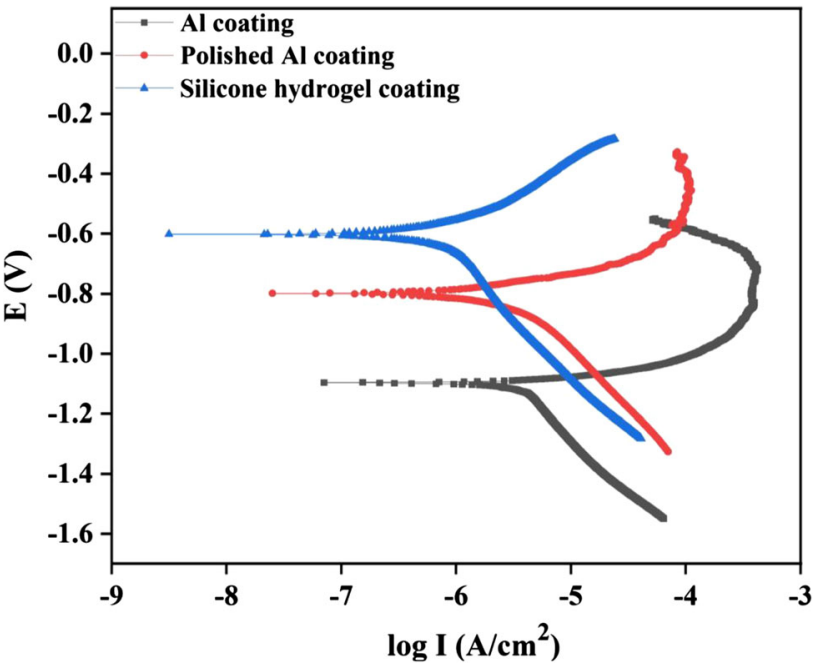

Fig. 11 Tafel curve of the Al coating, the polished $\mathrm{Al}$ coating and the silicone hydrogel coating.

coating surfaces. The algae adhesion rate of the Al coating is higher than those of the polished $\mathrm{Al}$ coating, and silicone hydrogel coating. The adhesion rate of the Al coating after 
Table 1 Potentiodynamic polarization parameters of the coatings immersed in ASW

\begin{tabular}{lcccc}
\hline \multicolumn{1}{c}{ Samples } & $E_{\text {corr }}(\mathrm{V})$ & $I_{\text {corr }}\left(\mathrm{A} / \mathrm{cm}^{2}\right)$ & $\boldsymbol{\beta}_{\boldsymbol{c}}(\mathrm{V} / \mathrm{dec})$ & $\boldsymbol{\beta}_{\boldsymbol{a}}(\mathrm{V} / \mathrm{dec})$ \\
\hline Al coating & -1.0135 & $9.6756 \times 10^{-6}$ & 0.20274 & 0.20192 \\
Polished Al coating & -0.8279 & $2.3407 \times 10^{-6}$ & 0.30651 & 0.07761 \\
Hydrogel coating & -0.7838 & $8.6252 \times 10^{-7}$ & 0.49709 & 0.22707 \\
\hline
\end{tabular}

immersing in the algae suspension for 7 days reached $14.39 \%$. Compared with the $\mathrm{Al}$ coating, the adhesion rate of the polished Al coating and silicone hydrogel coating dropped to $11.16 \%$ and $4.88 \%$, respectively. In other words, the attachment rate of the polished Al coating and silicone hydrogel coating reduced by $22.45 \%$ and $66.09 \%$ than that of the Al coating. The silicone hydrogel coating exhibited much better antifouling properties. This is consistent with Brady's research (Ref 17) that low elastic modulus and surface energy are not conducive to the attachment of fouling organisms. The surface energy of the silicone hydrogel measured by the contact angle is 21.34 $\mathrm{mJ} / \mathrm{m}^{2}$ and the elastic modulus is $8.34 \mathrm{kPa}$. The algae adhesion rate of the polished $\mathrm{Al}$ coating is lower than that of the Al coating because the surface of the polished $\mathrm{Al}$ coating is smoother, which is not conducive to microorganism's attachment (Ref 44).

Figure 11 shows the Tafel curve of the Al coating, the polished Al coating and the silicone hydrogel coating. Tafel curves are extensively used in electrochemistry, particularly in the investigation of corrosion (Ref 45, 46). The Tafel curve can be used to determine the corrosion potential. Corrosion potential $\left(E_{\text {corr }}\right)$, corrosion current $\left(I_{\text {corr }}\right)$, cathodic Tafel slope $\left(\beta_{c}\right)$ and anodic Tafel slope $\left(\beta_{a}\right)$ are listed in Table 1. The electrochemical results show that the corrosion potentials $\left(E_{c o r r}\right)$ of the Al coating, polished $\mathrm{Al}$ coating, and silicone hydrogel coating are $-1.0135 \mathrm{~V}$, $-0.8279 \mathrm{~V}$, and $-0.7838 \mathrm{~V}$, respectively. Additionally, corrosion current density of the three coatings are $9.6756 \times 10^{-6} \mathrm{~A} / \mathrm{cm}^{2}, 2.3407 \times 10^{-6} \mathrm{~A} / \mathrm{cm}^{2}$, and $8.6252 \times 10^{-7}$ $\mathrm{A} / \mathrm{cm}^{2}$, respectively. It is worth noting that the corrosion resistance of polished $\mathrm{Al}$ is between the silicone hydrogel coating and the Al coating, indicating that hydrogel materials have a positive effect on anti-corrosion performance. This could be explained that silicone hydrogel decreased the porosity by brushing on the porous coating and acted as a barrier coating (Ref 47 ). The polished Al coating has a smooth surface and it is easier to form a stable and uniform oxide film, so its anti-corrosion performance is higher than $\mathrm{Al}$ coating (Ref 48). Compared with the previous research (Ref 23-25) on the hydrogel coatings prepared by chemical methods, the arc spraying method does not require complex reagents and strict reaction conditions, and more importantly, the arc spraying technique has great advantages in terms of cost efficiency and large-scale fabrication.

\section{Conclusions}

Porous aluminum coatings were successfully fabricated by cored wire arc spraying, and the size of the pores can be controlled by regulating the size of the cored powders of sodium chloride. An antifouling layer, silicone hydrogel, was further deposited on the porous aluminum coating. Compared with the weak adhesion of hydrogel on the nonporous $\mathrm{Al}$ coating, the hydrogel penetrated into the open pores of the porous aluminum coatings, and the porous aluminum structure significantly improved the adhesion of the silicone hydrogel. In addition, the results of adhesion tests of algae and electrochemical tests suggest that the presence of silicone hydrogel coating significantly enhanced the corrosion resistance and antifouling properties. The silicone hydrogel plays a role of barrier and antifouling, thereby slowing down corrosion and achieving antifouling performance. The results provide a promising way of preparing multifunctional coatings for protection of marine infrastructures.

Acknowledgments This work was supported by the K.C. Wong Education Foundation (grant \# GJTD-2019-13), Ningbo 3315 Talents Program (grant \# 2020A-29-G), and Chinese Academy of Sciences President's International Fellowship Initiative (grant \# 2020VEA0005).

\section{References}

1. SE Coetser TE Cloete 2005 Biofouling and Biocorrosion in Industrial Water Systems Crit. Rev. Microbiol 314213232

2. LY Xie F Hong CX He CF Ma JH Liu GZ Zhang C Wu 2011 Coatings with a Self-Generating Hydrogel Surface for Antifouling Polymer 521737383744

3. I Fitridge T Dempstera J Guenther R Nys de 2012 The Impact and Control of Biofouling in Marine Aquaculture: A Review Biofouling 287649669

4. GC Ling MH Low M Erken S Longford S Nielsen AJ Poole P Steinberg D McDougald S Kjelleberg 2014 Micro-Fabricated Polydimethyl Siloxane (PDMS) Surfaces Regulate the Development of Marine Microbial Biofilm Communities Biofouling 303 323335

5. G Scandura R Ciriminna YJ Xu M Pagliaro G Palmisano 2016 Nanoflower-Like Bi2WO6 Encapsulated in ORMOSIL as a Novel Photocatalytic Antifouling and Foul-Release Coating Chem. Eur. J. 222170637067

6. M Thouvenin JJ Peron C Charreteur P Guerin JY Langlois K Vallee-Rehel 2002 A Study of the Biocide Release from Antifouling Paints Prog. Org. Coat 4427583

7. I Banerjee RC Pangule RS Kane 2011 Antifouling Coatings: Recent Developments in the Design of Surfaces That Prevent 
Fouling by Proteins, Bacteria, and Marine Organisms Adv. Mater. 236690718

8. A.G. Nurioglu A.C.C. Esteves, and G.D. With, Non-BiocideRelease Antifouling Coatings Based on Molecular Structure Design for Marine Applications, J. Mater. Chem. B, 2015, 3(32), p 6547-6570.

9. Y. Bhoj, M. Tharmavaram, and D. Rawtani, A Comprehensive Approach to Antifouling Strategies in Desalination, Marine Environment, and Wastewater Treatment, Chem. Phys. Impact, 2021, 2, p 100008.

10. J Cong XM Zhang KS Chen J Xu 2002 Fiber Optic Bragg Grating Sensor Based on Hydrogels for Measuring Salinity Sensor Actuat. B. Chem. 873487490

11. J Wang S Banerji N Menegazzo W Peng QJ Zoua KS Booksh 2011 Glucose Detection with Surface Plasmon Resonance Spectroscopy and Molecularly Imprinted Hydrogel Coatings Talanta 86133141

12. H Cheng K Yue M Kazemzadeh-Narbat YH Liu A Khalilpour BY Li YS Zhang N Annabi A Khademhosseini 2017 MusselInspired Multifunctional Hydrogel Coating for Prevention of Infections and Enhanced Osteogenesis ACS Appl. Mater. Inter. 9 41142811439

13. M Fischer M Vahdatzadeh R Konradi J Friedrichs MF Maitz U Freudenberg C Werner 2015 Multilayer Hydrogel Coatings to Combine Hemocompatibility and Antimicrobial Activity Biomaterials 56198205

14. Y Nakayama KJ Youn S Nishi H Ueno T Matsuda 2015 Development of High-Performance Stent: Gelatinous PhotogelCoated Stent That Permits Drug Delivery and Gene Transfer J. Biomed. Mater. Res. 574559566

15. T Ekblad G Bergström T Ederth SL Conlan R Mutton AS Clare S Wang YL Liu Q Zhao F D'Souza GT Donnelly PR Willemsen ME Pettitt ME Callow JA Callow B Liedberg 2008 Poly(Ethylene Glycol)-Containing Hydrogel Surfaces for Antifouling Applications in Marine and Freshwater Environments Biomacromol 91027752783

16. HW Zhu JN Zhang P Su TQ Liu CC He DQ Feng HL Wang 2020 Strong Adhesion of Poly(Vinyl Alcohol)-Glycerol Hydrogels onto Metal Substrates for Marine Antifouling Applications Soft Matter 163709717

17. RF Brady IL Singer 2000 Mechanical Factors Favoring Release from Fouling Release Coatings Biofouling 1517381

18. Q.Y. Xie J.S. Pan, C.F. Ma, and G.Z. Zhang, Dynamic Surface Antifouling: Mechanism and Systems, Soft Matter, 2019, 15(6), p 1087-1107.

19. C Bressy M Lejars 2014 Marine Fouling : An Overview Marine Fouling J. Ocean Technol. 91928

20. RE Baier 2006 Surface Behaviour of Biomaterials: The Theta Surface for Biocompatibility J. Mater. Sci. Mater. M. 17111057 1062

21. P Hu QY Xie CF Ma GZ Zhang 2020 Silicone-Based FoulingRelease Coatings for Marine Antifouling Langmuir 3692170 2183

22. W Zhang RX Wang ZM Sun XW Zhu Q Zhao TF Zhang A Cholewinski F Yang BX Zhao R Pinnaratip PK Forooshanie BP Lee 2020 Catechol-Functionalized Hydrogels: Biomimetic Design, Adhesion Mechanism, and Biomedical Applications Chem. Soc. Rev. 492433464

23. G Westwood TN Horton JJ Wilker 2007 Simplified Polymer Mimics of Cross-Linking Adhesive Proteins Macromolecules 40 1139603964

24. J.P. Yang, B. Xue, Y.Y. Zhou, M. Qin, W. Wang, and Y. Cao, Spray-Painted Hydrogel Coating for Marine Antifouling, Adv. Mater. Technol., 2021, 6(3), p 2000911.
25. Y. Gao, J.J. Chen, X.Y. Han, Y.D. Pan, P.Y. Wang, T.J. Wang, and T.Q. Lu, A Universal Strategy for Tough Adhesion of Wet Soft Material, Adv. Funct. Mater., 2020, 30(36), p 2003207.

26. CW Jennings 1972 Surface Roughness and Bond Strength of Adhesives J. Adhesion 412538

27. FH Su K Yao 2014 Facile Fabrication of Superhydrophobic Surface with Excellent Mechanical Abrasion and Corrosion Resistance on Copper Substrate by A Novel Method ACS Appl. Mater. Inter. 61187628770

28. PH Pi W Mu G Fei YL Deng 2013 Superhydrophobic Film Fabricated by Controlled Microphase Separation of PEO-PLA Mixture and Its Transparence Property Appl. Surf. Sci. 273184 191

29. XJ Wang SQ Xu SW Zhou W Xu M Leary P Choong M Qian M Brandt YM Xie 2016 Topological Design and Additive Manufacturing of Porous Metals for Bone Scaffolds and Orthopaedic Implants: A Review Biomaterials 83127141

30. JT Yao JQ Ren HB Huo GJ Yang CX Li CJ Li 2014 Deposition Behavior of Semi-Molten Spray Particles During Flame Spraying of Porous Metal Alloy J. Therm. Spray. Technol. 236991999

31. CJ Li J Zou HB Huo JT Yao GJ Yang 2015 Microstructure and Properties of Porous Abradable Alumina Coatings FlameSprayed with Semi-Molten Particles J. Therm. Spray. Technol. 25 119

32. BH Toby 2001 EXPGUI, a Graphical User Interface for GSAS J. Appl. Crystallogr. 342210213

33. "Standard Test Method for Adhesion or Cohesion Strength of Thermal Spray Coatings", C633-01, ASTM, 2001.

34. DK Owens RC Wendt 1969 Estimation of the Surface Free Energy of Polymers J. Appl. Polym. Sci. 1317411747

35. EC Muniz G Geuskens 2001 Compressive Elastic Modulus of Polyacrylamide Hydrogels and Semi-IPNs with Poly(N-isopropylacrylamide) Macromolecules 341344804484

36. Y. Tian, H.J. Zhang, X.Y. Chen, A. McDonald, S.J. Wu, T.H. Xiao, and H. Li, Effect of Cavitation on Corrosion Behavior of HVOF-Sprayed WC-10Co4Cr Coating with Post-sealing in Artificial Seawater, Surf. Coat. Tech., 2020, 397, p 126012.

37. XL Zhang ZH Jiang ZP Yao Y Song ZD Wu 2009 Effects of Scan Rate on the Potentiodynamic Polarization Curve Obtained to Determine the Tafel Slopes and Corrosion Current Density Corros. Sci. 513581587

38. H.S. Lee, J.K. Singh, and J.H. Park, Pore Blocking Characteristics of Corrosion Products formed on Aluminum Coating Produced by Arc Thermal Metal Spray Process in $3.5 \mathrm{wt} . \% \mathrm{NaCl}$ Solution, Constr. Build. Mater., 2016, 113, p 905-916.

39. JX Zhou MC Yang RQ Wang XM Pang 2017 Annealing Behavior of Aluminum Coating Prepared by Arc Spraying on P355NL1 Steel Surf. Coat. Tech. 3305350

40. A. E. Erfan, S. Hamidreza, G. Mohamad A., M. Javad, P. Larry, Study of Corrosion Behavior of Arc Sprayed Aluminum Coating on Mild Steel, J. Therm. Spray. Technol., 2012, 21(6), p 11951202.

41. J Wilden JP Bergmann S Jahn S Knapp F Rodijnen van G Fischer 2007 Investigation about the Chrome Steel Wire Arc Spray Process and the Resulting Coating Properties J. Therm. Spray. Technol. 16 5-6 759767

42. W Tillmann E Vogli M Abdulgader M Gurris D Kuzmin S Turek 2008 Particle Behavior During the Arc Spraying Process with Cored Wires J. Therm. Spray. Technol. 17966973

43. J.W. Yang, R.B. Bai, B.H. Chen, and Z.G. Suo, Hydrogel Adhesion: A Supramolecular Synergy of Chemistry, Topology, and Mechanics, Adv. Funct. Mater., 2019, 30(2), p 1901693.

44. M Alnnasouri C Lemaitre C Gentric C Dagot MN Pons 2011 Influence of Surface Topography on Biofilm Development: Experiment and Modeling Biochem. Eng. J. 153845 
45. X.X. Wang, H. Zhao, S.Z. Wu, X.K. Suo, X.C. Wei, and H. Li, Aluminum-Polyethylene Composite Coatings with Self-Sealing Induced Anti-Corrosion Performances, J. Mater. Process. Tech., 2020, 282, p 116642.

46. LL Liu J Xu P Munroe JK Xu ZH Xie 2014 Electrochemical Behavior of $\left(\mathrm{Ti}_{1-\mathrm{x}} \mathrm{Nb}_{\mathrm{x}}\right)_{5} \mathrm{Si}_{3}$ Nanocrystalline Films in Simulated Physiological Media Acta Biomater. 10210051013

47. PA Sørensen S Kiil K Dam-Johansen CE Weinell 2009 Anticorrosive Coatings: A Review J. Coat. Technol. Res. 62135176
48. T Suter Y Müller P Schmutz OV Trzebiatowski 2005 Microelectrochemical Studies of Pit Initiation on High Purity and Ultra High Purity Aluminum Adv. Eng. Mater. 75339348

Publisher's Note Springer Nature remains neutral with regard to jurisdictional claims in published maps and institutional affiliations. 